OPEN ACCESS

Edited by:

Christel Bidet-Ildei, University of Poitiers, France

Reviewed by:

Qian Cui,

University of Electronic Science and Technology of China, China

Yi Lei,

Shenzhen University, China

${ }^{*}$ Correspondence:

Xiao Gao

gaoxiaox@swu.edu.cn

tThese authors have contributed equally to this work and share first authorship

Specialty section: This article was submitted to

Emotion Science,

a section of the journal

Frontiers in Psychology

Received: 10 July 2019 Accepted: 05 November 2019 Published: 29 November 2019

Citation

So MY, Wang X and Gao X (2019) Body Dissatisfaction Enhances

Awareness and Facilitates the Consolidation of Body-Related Words During Rapid Serial Visual

Presentation

Front. Psychol. 10:2614. doi: 10.3389/fpsyg.2019.02614

\section{Body Dissatisfaction Enhances Awareness and Facilitates the Consolidation of Body-Related Words During Rapid Serial Visual Presentation}

\author{
Man Yi So ${ }^{1,2+}$, Xinyu Wang ${ }^{1,2+}$ and Xiao Gao ${ }^{1,2 *}$ \\ ${ }^{1}$ Key Laboratory of Cognition and Personality, Ministry of Education, Southwest University, Chongqing, China, ${ }^{2}$ Faculty \\ of Psychology, Southwest University, Chongqing, China
}

Attentional biases have received considerable focus in research on cognitive biases and body dissatisfaction (BD). However, most work has focused on spatial allocation of attention. The current two experiments employed a rapid serial visual presentation (RSVP) task to investigate attention bias to body-related words in the temporal domain among young females with high and low BD. During this task, there were two targets presented in the same stimulus stream. The first target was defined as target one (T1) and the second was defined as target 2 (T2). Participants were asked to identify T2 while ignoring $\mathrm{T} 1$ in single task mode or identify both targets in the dual task mode. In the current study, Experiment 1 assessed the stimulus-driven attention of body-related stimuli. Participants were required to identify a target of neutral word (T2) as quickly and accurately as possible while ignoring the preceding target (T1) of neutral, fat-, or thinrelated words. As expected, we observed spontaneous attentional blink (AB) effects elicited by both fat- and thin-related T1s among participants with high $\mathrm{BD}$, suggesting enhanced awareness of body-related stimuli even when this information does not have to be identified. Such effects did not emerge among participants without BD. Experimental 2 investigated the goal-directed attention of body-related stimuli, during which participants needed to identify both the T1 and neutral T2. Participants with BD showed reduced $A B$ effects after both fat- and thin-related $T 1$, suggesting facilitated consolidation of body-related information in goal-directed attention among participants with $\mathrm{BD}$. These findings have important clinical implications that it provided insight for creating more accurate attention bias modification (ABM) task aiming at reducing and preventing $\mathrm{BD}$ among young females.

Keywords: attentional blink, temporal attention, attentional bias, body dissatisfaction, anxiety

\section{INTRODUCTION}

Body dissatisfaction (BD) is one of the prominent risk and maintenance factors for eating disorders (EDs; Stice and Shaw, 2002; Stice et al., 2010). BD is very common among young women across countries and cultures. For example, approximately $40-50 \%$ of women in the United States feel dissatisfaction with their body (Bearman et al., 2006). Fifty percent of adult women in Iceland 
(Matthiasdottir et al., 2010) and 48.3\% of women in Germany (von Lengerke et al., 2012) report BD, and the proportion in China is similar to those in Western societies (Chen, 2006).

There is abundant literature on information processing preferences related to body size and shape, most of which have suggested that attentional biases toward body-related cues contribute to the etiology and maintenance of $\mathrm{BD}$ and EDs (Cash and Strachan, 2002; Dobson and Dozois, 2004; Lee and Shafran, 2004). Vitousek and Hollon (1990) first proposed a cognitive model of EDs to explain why and how patients with EDs developed biased cognitive processing of body-related information (Vitousek and Hollon, 1990). Later, other cognitive theories of $\mathrm{BD}$ suggested that schemas related to appearance, shape, and weight influence processing of body image information (Cash and Labarge, 1996; Williamson et al., 2004).

Attentional biases have received the greatest amount of focus among research on cognitive biases and BD. Early studies often used modified Stroop color naming tasks to study attentional bias in ED samples, and most of the results indicated that ED samples were often slower to name the color of disorder-relevant stimuli than the neutral cues (Perpina et al., 1998; Sackville et al., 1998; Davidson and Wright, 2002; Lee and Shafran, 2004). Later, researchers adopted the dot probe task to investigate attentional bias in EDs or BD. In this task, a pair of stimuli (e.g., a bodyrelated stimulus and a neutral stimulus) are briefly presented on a screen, one above the other or one beside the other, and the pair of stimuli are immediately followed by a dot (the probe) in the location of one of the stimuli. Participants are required to respond to the probe as quickly and accurately as possible.

A recent literature has reviewed 22 studies exploring attention biases associated with BD (for review, see Rodgers and DuBois, 2016). It reported that studies employing a dot-probe paradigm found that when compared to individuals with low $\mathrm{BD}$, individuals with high BD showed a greater attentional bias toward negative appearance-related (Rosser et al., 2010; Gao et al., 2011) as well as positive appearance-related stimuli (Glauert et al., 2010; Rosser et al., 2010; Onden-Lim et al., 2012). Studies using eye tracking measurements have reported that relative to a low $\mathrm{BD}$ group, women with BD tend to orient more attention toward negative appearance-related stimuli (Gao et al., 2011, 2012, 2014). However, some researchers have reported attentional avoidance of appearance-related information in the late attentional process phase (Glauert et al., 2010; Gao et al., 2012).

Several studies using various paradigms have demonstrated that attentional biases toward body-related information are associated with BD. However, most have focused on the spatial allocation of attention to body-related stimuli. Some used a dot probe paradigm (i.e., Glauert et al., 2010; Gao et al., 2011, 2012), some employed a visual search task (i.e., Smeets et al., 2011; Gao et al., 2014), while others utilized a spatial cueing paradigm (i.e., Gao et al., 2013). In all the above tasks, subcomponents of attentional bias including orientation, engagement, disengagement, and maintenance could be investigated using different experimental manipulations combining with reaction time. However, the attentional bias in the spatial domain is not the only mechanism of interest for understanding the cognitive processing of body-related information in terms of its association with BD. Below are some limitations in previous studies in this field.

First, few studies have distinguished two important components of attention, namely, goal-directed and stimulusdriven attention (Corbetta and Shulman, 2002). The former is top-down controlled attention (i.e., it focuses on relevant signals derived from task demands; Annic et al., 2016), whereas the latter is bottom-up driven attention (i.e., it is captured by salient properties of stimuli that are usually irrelevant with the task; Desimone and Duncan, 1995). The tasks used in previous studies - for example, the dot probe task and the visual search task - capture both elements of attention. However, they can't distinguish those two components. For example, there is stimulus-driven attention in the dot-probe task, such that body-related cues could automatically capture attention among participants concerned with their body weight or shape (e.g., Gao et al., 2011). Meanwhile, there is also an element of goal-directed attention, such that participants must use attentional control to overcome the interference of body-related cues to identify the target (e.g., arrow direction or dot location).

Second, previous studies have showed that individuals with $\mathrm{BD}$ are more likely to provide fat-related judgments of ambiguous information (Chen and Jackson, 2005), more prone to interpret fat-related stimuli in a negative way (Chen, 2006), orient their attention quicker to body-related stimuli rather than other cues (Gao et al., 2011, 2012, 2014), and maintained more attention to fat-related stimuli (Gao et al., 2011). These findings showed increased recruitment of stimulus-driven processes, which may involve considerable attentional resources. However, previous literature focusing on the spatial domain could not determine whether the attentional orientation, engagement, or maintenance bias toward body-related information occupied more attentional resources when the location of the critical stimuli was attended. This is critical for the onset and maintenance of BD and even EDs, because excessive attention to body-related information may reflect deficits in goal-directed attentional resources, resulting in ruminating or dwelling on the negative body-related information.

Third, few studies have directly examined the attentional interference in the temporal domain among people with BD. For example, previous studies could not determine whether body-related information is more easily captured than neutral stimuli among people with BD when processing flows of information in the temporal domain. In addition, research on spatial attentional bias has reported that when body-related information is no longer present, people with $\mathrm{BD}$ showed delayed spatial disengagement from the location where the critical stimuli were presented (Gao et al., 2011, 2013). Thus, we might ask whether processing body-related information influences the processing of subsequent stimuli. Investigating body-related information processing in the temporal domain is critical to understanding how cognitive characteristics associated with BD contribute to dynamic alterations regarding what information reaches conscious awareness. This may then lead to a better understanding of how to intervene and prevent BD (Browning et al., 2010; Van Dam et al., 2012).

One common approach of examining the nature of human attentional processing in the temporal domain is rapid serial 
visual presentation (RSVP), which was first developed by Raymond (Raymond et al., 1992). In this task, stimuli appear in rapid succession in the same spatial location, at rates ranging from 8-16 stimuli per second, and the stream of stimuli typically contains 6-20 items. In the single-task mode, participants are required to identify a specific target in the distractor stream. For example, they are asked to identify a letter $X$ in a numeric stream, or identify a white letter in a red-letter-stream. Detection of the target usually occurs with approximately 95\% accuracy (e.g., Shapiro et al., 1997). In the dual-task mode, participants are asked to identify the first target (T1) as well as the second target (T2), which are presented in the same stimulus stream. Relative to the single task, in the dual-task mode, accuracy of $\mathrm{T} 2$ detection in a 100 - to $500-\mathrm{ms}$ window after T1 is decreased following T1 identification (e.g., Martin and Shapiro, 2008). The decrease in performance in the dual-task RSVP paradigm relative to the single-task paradigm is called an attentional blink $(\mathrm{AB})$. The $\mathrm{AB}$ exhibits a $\mathrm{U}$-shaped return to optimal $\mathrm{T} 2$ detection rates as the number of intervening stimuli increases (Van Dam et al., 2012).

Rapid serial visual presentation paradigms are useful for exploring the temporal mechanisms of attention as modulated by emotion (McHugo et al., 2013; for review, see Mishra et al., 2017). On the one hand, the stimulus-driven bottom-up engagement of attention could be assessed using emotional T1s, which do not have to be identified, in the single task mode. For example, the presentation of emotional $\mathrm{T} 1 \mathrm{~s}$ in a single task (for which a response is not required) hinders the identification of a subsequent T2 at short temporal lags, producing a phenomenon termed emotion-induced attentional blindness (EAB, Most et al., 2005; McHugo et al., 2013). Thus, it seems that emotional T1s automatically capture attention, occupying limited visual working memory resources, and hence compromising processing of the following T2. On the other hand, goal-directed, topdown engagement of attention could also be investigated using emotional T1s in the dual-task mode. For example, emotional T1s can increase blink magnitude (greater reduction in T2 response accuracy) at short lags compared to neutral T1s (de Jong et al., 2010; for review, see Mishra et al., 2017).

Rapid serial visual presentation has also been employed to investigate specific mechanisms of attention that are biased or impaired in a particular population (Bredemeier et al., 2011; Van Dam et al., 2012; Weierich and Treat, 2015). Previous studies have used $\mathrm{EAB}$ to measure the extent to which individuals with anxiety disorders exhibit increased attentional capture by or difficulty disengaging from - concern-relevant stimuli (e.g., Olatunji et al., 2011). Other studies using symptom-related T1s in the dual-task mode observed reduced length of $A B$ among participants with high levels of arachnophobia symptoms (Cisler et al., 2007) as well as with high levels of PTSD symptoms (Amir et al., 2009), suggesting that individuals with anxiety about spiders or PTSD symptoms may require fewer attentional resources to process symptom-related stimuli when employing goal-directed attention.

Giving the limitations of previous studies assessing the BD and attention interaction, the current two experiments employed RSVP to investigate the attentional bias to fat- and thin-related words in the temporal domain among young females with high and low BD. Experiment 1 aimed to assess the stimulusdriven attention to body-related stimuli by employing neutral, fat-, and thin-related T1s in the single-task RSVP paradigm. Participants were required to identify a neutral T2 as precisely as possible while ignoring the preceding T1. Experiment 2, by using a dual-task RSVP paradigm, sought to investigate goal-directed attention to body-related stimuli by using the same T1 as in Experiment 1. Participants needed to identify both the T1 and neutral T2 accurately. According to Vitousek and Hollon's (1990) cognitive theory of EDs, attentional biases toward weight-related information arise as a result of underlying maladaptive schemata associated with shape, weight and self. Individuals with maladaptive schemata differ from those without in several ways, including enhanced attention to and more efficient processing of schema-related information. Based on Vitousek and Hollon's (1990) cognitive theory of EDs and previous literature (Glauert et al., 2010; Gao et al., 2011, 2013), we expected that (1) there would be a spontaneous $A B$ effect after both fat- and thin-related T1s in the single-task RSVP (Experiment 1) among participants with high BD. These spontaneous $\mathrm{AB}$ effects, on the other hand, would be weaker or non-existent among participants with low $\mathrm{BD}$; and (2) compared to the low $\mathrm{BD}$ group, the $\mathrm{AB}$ effects induced by both fat- and thin-related T1s in the dual-task RSVP (Experiment 2) would be less pronounced in the high $\mathrm{BD}$ group, leading to a higher accuracy rate for $\mathrm{T} 2 \mathrm{~s}$ after both fat- and thin-related $\mathrm{T} 1 \mathrm{~s}$ in a short time window.

\section{EXPERIMENT 1}

In Experiment 1, our main objective was to examine the interaction of $\mathrm{BD}$ and stimulus-driven attention, which may be characterized by automatic attention capture as a result of bodyrelated stimuli among women with high BD. Single-task RSVP was employed with neutral (household words), fat-, and thinrelated words as T1 and affectively neutral words as T2 (words referring to instruments). The T2 appeared anywhere from the first (lag 1) to the sixth position (lag 6) after T1. Participants were required to detect the $\mathrm{T} 2$ as accurately as possible while ignoring other distractors, including the T1. As described in the central capacity-limited model (Chun and Potter, 1995), the to-be-ignored $\mathrm{T} 1$ would receive consolidation processing and occupy considerable working memory resources if it is of high salience, which would in turn interfere with T2 identification. Thus, according to this model (Chun and Potter, 1995) and based on previous findings (Glauert et al., 2010; Gao et al., 2011, 2013), we hypothesized that women with high BD would show spontaneous $\mathrm{AB}$ effects in a short period after both fat- and thinrelated $\mathrm{T} 1 \mathrm{~s}$, while these $\mathrm{AB}$ effects would be weak or non-existent in the low BD group.

\section{Method Participants}

The sample included 57 young women drawn from undergraduate classes at Southwest University, Chongqing, 
TABLE 1 | Demographic information of participants in Experiment 1 and Experiment 2.

\begin{tabular}{lccrr}
\hline & High BD group & Low BD group & $\boldsymbol{t}$ & \multicolumn{1}{c}{$\boldsymbol{p}$} \\
\hline Experiment 1 & 28 & & & \\
$N$ & $20.86(1.84)$ & $21.52(1.27)$ & -1.58 & 0.120 \\
Age (years) & $20.71(2.77)$ & $19.43(1.59)$ & 2.13 & 0.037 \\
BMl (kg/m $\left.{ }^{2}\right)$ & $2.84(0.27)$ & $1.04(0.37)$ & 20.98 & $<0.001$ \\
NPS-F & $2.03(0.49)$ & $1.86(0.53)$ & 1.23 & 0.224 \\
STAl-Trait subscale & 30 & & & \\
Experiment 2 & $22.60(2.50)$ & $22.17(1.15)$ & 0.86 & 0.392 \\
N & $20.31(2.15)$ & $19.14(1.52)$ & 2.43 & 0.018 \\
Age (years) & $2.82(0.27)$ & $1.31(0.62)$ & 12.24 & $<0.001$ \\
BMl (kg/m ${ }^{2}$ ) & $2.27(0.78)$ & $2.01(0.47)$ & 1.60 & 0.116 \\
NPS-F & & & & \\
STAl-trait subscale & & & & \\
\hline NPS-F = negative physical self scale-fatness; STAl-Trait subscale = the state-trait \\
anxiety inventory-trait subscale.
\end{tabular}

China. Twenty-eight of them were in the high BD group and 29 were in the low $\mathrm{BD}$ group. The entire sample ranged from 18 to 25 years of age $(M=21.19, \mathrm{SD}=1.60)$, and their BMIs ranged from 16.23 to $28.40(M=20.06, \mathrm{SD}=2.31)$. The demographic information and self-reported data of the two groups are presented in Table 1. All were right-handed non-smokers, with no current or previous neurological or psychiatric illness, had a normal or corrected-to-normal vision, and had a normal color vision as assessed by several basic color tests.

Following ethics approval from Southwest University, participants were recruited via on-campus advertisements. Subsequently, 338 female undergraduate students engaged in the first session of the study. After reading a general overview of the study and signing informed consent, participants completed individually administered self-reported measures of age, handedness, history of neurological or psychiatric illness, the Negative Physical Self Scale-Fatness (NPS-F), and the State-Trait Anxiety Inventory-Trait subscale (STAI-Trait subscale). Objective weight and height measurements were taken and a vision test was completed. Appointments were made with 30 women randomly selected from subgroups who scored higher than 2.5 and with 30 women who scored lower than 1.5 on the NPS-F. With the aim of obtaining extreme groups, the current cutoffs rather than the median were chosen. These cutoffs are based on previous investigations in a sample $(n=2152)$ drawn from middle schools, high schools, and colleges/universities in China ranging from 12 to 24 years of age, where the mean score on the NPS-F was $1.06(\mathrm{SD}=0.90)$, and the mean score for females $(n=1242)$ was about 1.3 [see Figure 1 in Chen et al. (2006)]. Using these cutoffs, previous studies found robust group differences for attentional biases to body-related information (Gao et al., 2011, 2012, 2014). After completing these measures, participants took part in the experimental task session. Two participants in high BD group and 1 in the low BD group did not finish the experimental task. Thus, the final sample consisted of 57 participants.

\section{Measures}

\section{Demographics}

Participants' age and grade in university were assessed. BMI was calculated from objectively measured height and weight $\left[\right.$ BMI $=$ Weight $(\mathrm{kg}) /$ Height $\left.^{2}\left(\mathrm{~m}^{2}\right)\right]$.

\section{Negative physical self scale-fatness subscale (NPS-F, Chen et al., 2006)}

The 11-item Fatness subscale of Negative Physical Self Scale measuring thoughts, feelings, and behaviors related to body weight dissatisfaction was used to assess BD. Each item was rated between $0=$ not at all like me and $4=$ very much like me. Sample items include "I am very distressed when I think about my weight," "I am fat in others' eyes," and "I have tried many ways to lose weight." Scores were obtained by summing the scores of all items and dividing this total by 11 to yield an average score ranging from 0 to 4 . Higher scores reflect a higher level of dissatisfaction. The NPS-F yields internally consistent scores $(\alpha=0.88)$, as well as stable scores over 3 weeks $(r=0.89)$ among female and male middle and high school students and undergraduates, and over 9 months $(r=0.70)$ among middle school and high school girls (Chen and Jackson, 2007). It has satisfactory convergent and predictive validity (Chen and Jackson, 2008) among samples of adolescents and young adults. Its alpha coefficient for the current study was $\alpha=0.87$.

\section{The state-trait anxiety inventory-trait subscale (stai-trait subscale) (Smith, 1972)}

STAI-Trait subscale is a brief self-rating scale assessing trait anxiety in adults. It consists of 20 items that evaluate how the respondent "generally" feels. The STAI-Trait subscale asks participants to rate the frequency of their feelings on a 4point Likert scale ranging from $1=$ almost never to $4=$ almost always. Sample items include "I am a steady person," "I lack self-confidence," and "I feel at ease." Scores were obtained by summing the scores of all items and dividing this total by 20 to yield an average score ranging from 1 to 4 with higher scores reflecting a higher level of trait anxiety. Its alpha coefficient for the current study was $\alpha=0.90$.

\section{Stimuli}

Fifty-one upright words [16 fat-related words, 16 thin-related words, 16 neutral household words, and 3 instrument-related words (Piano, Flute, and Erhu)] printed in white as targets and 39 inverted neutral household words printed in red as distractors were used. The 32 body related words were adapted from recent work (Gao, 2010; Gao et al., 2011). The word length and frequency were matched between the targets and the distractors. Twenty-seven female undergraduate students who did not participate in the formal experiment rated all bodyrelated words on valence (from $1=$ very negative to $9=$ very positive) and arousal (from $0=$ very peaceful to $8=$ very excited) on 9-point Likert scales. The fat- and thin-related words were rated to differ in valence, $F(1,53)=135.02, p<0.001$. Fat-related words $(M=2.14, \mathrm{SD}=1.15)$ were rated more negative than thinrelated words $(M=5.59, \mathrm{SD}=1.04)$. Fat-related words $(M=4.21$, $\mathrm{SD}=1.33)$ and thin-related words $(M=4.60, \mathrm{SD}=1.61)$ showed no differences in arousal ratings, $F(1,53)=0.95, p=0.334$. All 
the target words are listed in Appendix A with corresponding English translations.

\section{RSVP Task}

The RSVP procedure was programed with E-Prime 2.0 (Psychology Software Tools, Inc., Pittsburgh, PA, United States). At the beginning of the formal experiment, a white fixation and a blue fixation appeared successively in the middle of the screen and lasted for 500 and $300 \mathrm{~ms}$, respectively. Shortly thereafter, 13 distractors and 2 target stimuli were displayed successively, with each visible for $106 \mathrm{~ms}$ and no blank interval between each other. The distractors consisted of 13 inverted neutral household words printed in red. The T1 stimulus was one of the three conditions: one of (1) 16 fat-related words, (2) 16 thin-related words, or (3) 16 neutral household words. The T2 stimulus was one of the three instrument words. Each T1 and T2 stimulus was presented in an upright position and printed in white, with the same probability of occurrence. The T1 appeared randomly and equiprobably in the third to seventh position in the stimuli series, and the T2 appeared randomly and equiprobably from the first (lag 1) to the sixth (lag 6) position after T1. Other positions aside from T1 and T2 were filled with distractors. All distractors and targets were displayed in the center of the black background. This task was illustrated in Figure 1.

In single-task RSVP, participants were presented with a question about T2 ("Please identify the first upright word: Piano, Flute, Erhu, or I don't know”), while ignoring T1. The corresponding response key for each answer was counterbalanced across participants. In this task, participants were required to make judgments on T2 as accurately as possible. The question appeared until a response was made or for $5000 \mathrm{~ms}$ maximum. Participants would be led into the next trial after a 500-ms interval during which the screen remained black and blank.

\section{Procedures}

Before the experiment, participants were told that the current study aimed to determine human's visual reaction speed and would last for about $20 \mathrm{~min}$. Each participant completed a total of 648 trials of 18 conditions (three categories of T1 $\times 6 \mathrm{~T} 1-\mathrm{T} 2$ lags), which were randomly and equally presented in six blocks. Thus, each block consisted of 108 trials with each condition having six trials.

Individual testing sessions were scheduled between 8:30 and 11:30 am and 2:00-5:30 pm in a quiet soundproof room. Following the session, participants were debriefed about the research purposes and paid 30 Yuan as compensation for their time.

\section{Data Analysis}

T2 percent accuracy was analyzed using a 2 (Group: BD vs. Control $) \times 3$ (T1 category: Fat, Thin, and Neutral words $) \times 6$ (Lag: Lag 1-6) mixed model ANOVA. Then, for each category of T1, correlations were calculated between overall T2 percent accuracy and T2 percent accuracy at each lag with NPS-F and STAI-Trait subscale scores, respectively. Post hoc comparisons for significant main effects and interactions, as well as correlational analysis, used a Bonferroni correction to optimize power while minimizing Type I error rate. All statistics were computed using IBM SPSS Statistics 21.0.

\section{Results \\ Influence of BD on T2 Identification}

A 2 (Group: BD vs. Control) × 3 (T1 category: Fat, Thin, and Neutral words) $\times 6$ (Lag: Lag 1-6) mixed model ANOVA on T2 percent accuracy revealed a significant main effect of Lag, $F(5,275)=20.25, p<0.001$, partial $\eta^{2}=0.27$, with lower T2 accuracy at Lags 1 and 2 than at Lags 3-6. The main effect of Group was also significant, $F(1,55)=19.63, p<0.001$, partial $\eta^{2}=0.26$, reflecting better overall performance in the Control group $(M=0.94, S E=0.005)$ as compared to the high BD group $(M=0.91, S E=0.005)$. There were also significant interactions for Group $\times \operatorname{Lag}, F(5,275)=10.24, p<0.001$, partial $\eta^{2}=0.16$; Group $\times$ T1 category, $F(2,110)=3.98, p=0.023$, partial $\eta^{2}=0.07$; Lag $\times$ T1 category, $F(10,550)=3.43, p=0.001$, partial $\eta^{2}=0.06$; as well as a three-way interaction of Group $\times \mathrm{T} 1 \times \mathrm{Lag}, F(10$, $150)=2.83, p=0.007$, partial $\eta^{2}=0.05$.

Simple effect analysis was conducted based on the three-way interaction. Spontaneous AB effects were observed only in the high $\mathrm{BD}$ group when the T1s were fat- and thin-related words. T2 detection was significantly lower at Lags 1 and 2 than at Lags 3-6 following both fat- and thin-related T1s (all $p s<0.001$; Figure 2) in the high $\mathrm{BD}$ group. The low $\mathrm{BD}$ group did not show such $\mathrm{AB}$ effects. Meanwhile, the neutral T1 also elicited AB effects in the high $\mathrm{BD}$ group, such that T2 detection was worse at Lag 2 than at Lags 3-5 (all ps < 0.031). Comparisons between different T1 categories at different lags in the high BD group showed that T2 detection accuracy at Lag 1 after both fat- and thin-related T1s was lower as compared to those after neutral T1s (all $p s<0.001$ ), indicating that in the high $\mathrm{BD}$ group, the magnitudes of $\mathrm{AB}$ effects elicited by fat- and thin-related T1s were higher than were those elicited by neutral T1s at Lag 1 . The difference disappeared at later lags. However, there were no such spontaneous AB effects in the Control group (all $p s>0.099$ ). When comparing T2 detection between the two groups, T2 detection accuracy at Lags 1 and 2 after both fat- and thin-related T1s was significantly lower in the high BD group than it was in the Control group (all ps $>0.024$ ). No such difference was observed after neutral T1s.

\section{Correlation Between BD and T2 Identification}

Correlational analyses were computed across the two groups $(n=57)$. Using the Bonferroni correction, correlations were considered significant if the $p$-value was $<0.05 / 18=0.003$. NPS-F scores negatively correlated with T2 detection at Lag 1 following fat-related T1s $(r=-0.58, p<0.001)$, and T2 detection at both Lag $1(r=-0.51, p<0.001)$ and Lag $2(r=-0.38, p=0.003)$ following thin-related T1s (Figure 2B). Other correlations were not significant. STAI-Trait subscale scores did not correlate with $\mathrm{T} 2$ percent accuracy in any condition (all $p s>0.05$ ).

\section{Discussion}

Experiment 1 investigated stimulus-driven attention to bodyrelated stimuli by employing neutral, fat-, and thin-related T1 in a single-task RSVP paradigm. Participants were required to 


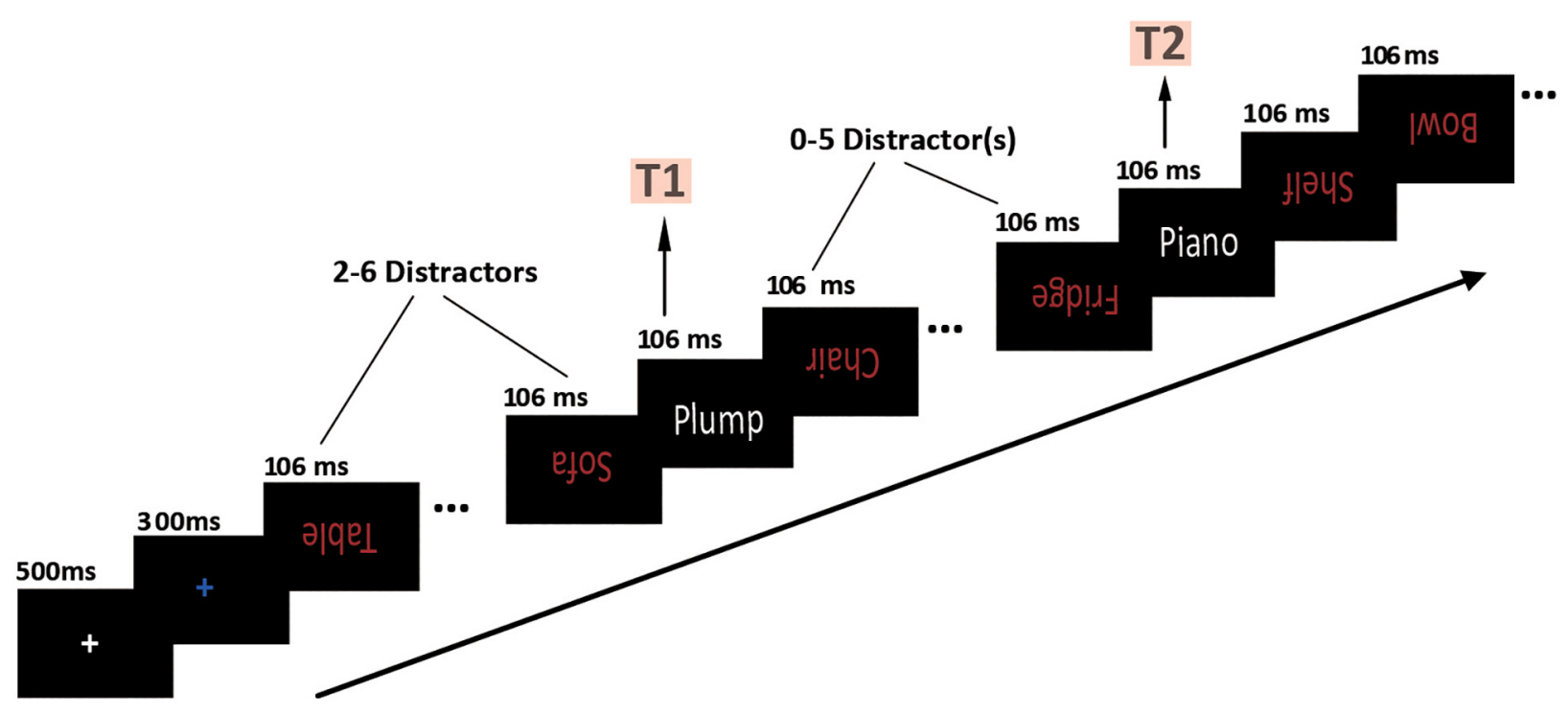

FIGURE 1 | Overview of a representative experimental trial.

\section{A}

B

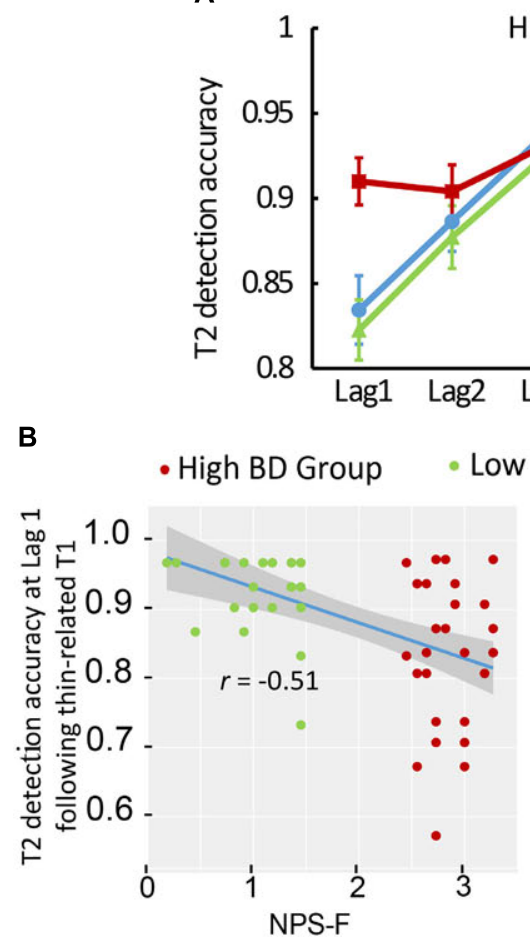

High BD Group

Low BD Group

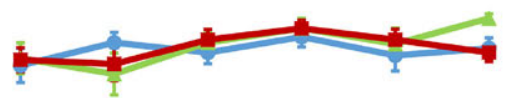

$-\mathrm{T}-\mathrm{T} 1$

$\pm \mathrm{F}-\mathrm{T} 1$

$\mathrm{N}-\mathrm{T} 1$

NPS-F=Negative Physical
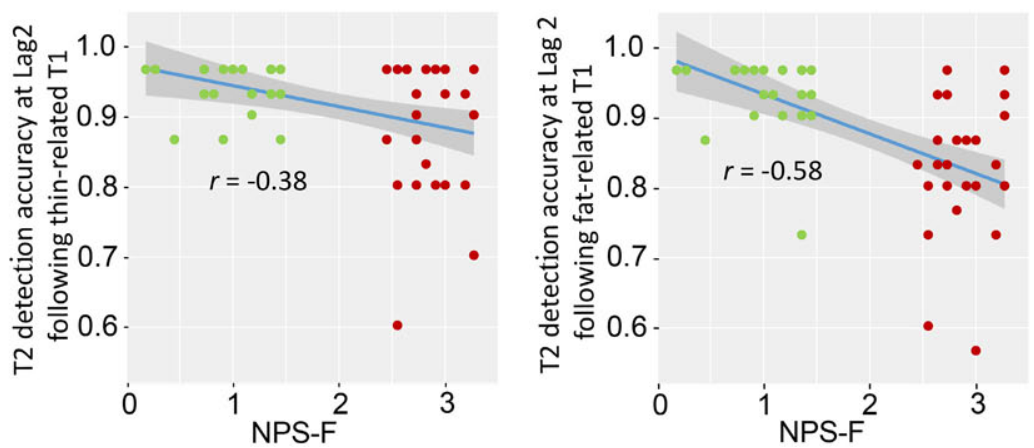

FIGURE 2 | Mean percentage of correct T2 identifications and correlation analysis in single task RSVP, Experiment 1. (A) Mean percentage of correct T2 identifications as a function of TI category and the lag between TI and T2. Error bars represent the standard error of the mean. (B) Correlation analysis between NPS-F scores and percentage of correct T2 identifications at Lag 1 following fat-related Tl, as well as at Lag 1 and Lag 2 following thin-related Tl, with 95\% confidence interval presented.

identify neutral $\mathrm{T} 2 \mathrm{~s}$ as accurately as possible while ignoring $\mathrm{T} 1 \mathrm{~s}$. As expected, we observed spontaneous $A B$ effects elicited by both fat- and thin-related T1s among participants with high
$\mathrm{BD}$, suggesting that body-related words capture their attention automatically even when the information is task-irrelevant. These effects did not emerge among participants without BD. 
When comparing the performance of the two groups in T2 identification, high BD group showed lower $\mathrm{T} 2$ response accuracy following both fat- and thin-related T1 during short temporal lags (106-212 ms). The mere presentation of body-related words produced a deficit in awareness of subsequent neutral stimuli at short temporal lags in the high $\mathrm{BD}$ group, showing an enhancement in stimulus-driven attention. These findings in the high BD group replicated observations from previous studies, where emotional stimuli were presented as to-be-ignored T1s in the RSVP stream while participants searched for a single target (Most et al., 2005; Mathewson et al., 2008; McHugo et al., 2013). The enhancement in stimulus-driven attention to both fat- and thin-related $\mathrm{T} 1 \mathrm{~s}$ in the high $\mathrm{BD}$ group is consistent with previous studies focusing on the spatial domain reporting speeded detection of body-related words (Gao et al., 2011) or pictures (Gao et al., 2012) among women with BD.

\section{EXPERIMENT 2}

As described in the Introduction, Experiment 2 aimed to investigate goal-directed attention to fat- and thin-related words in a dual-task RSVP paradigm. Participants were required to identify both T1 and T2 words as accurately as possible in an RSVP stream. Neutral, fat-, and thin-related words were again used as the three T1 categories, while neutral instrument words served as T2 words.

\section{Method}

Sixty young women drawn from undergraduate classes at Southwest University, Chongqing, China, 30 of whom were in the high $\mathrm{BD}$ group and 30 in the low $\mathrm{BD}$ group. The entire sample ranged from 18 to 25 years of age $(M=21.54, \mathrm{SD}=2.16)$, and their BMIs ranged from 16.02 to $26.17(M=19.73, \mathrm{SD}=1.99)$. All were right-handed non-smokers, with no current or previous neurological or psychiatric illness, had a normal or correctedto-normal vision, and had a normal color vision as assessed by several basic color tests. The demographic information and self-reported data of participants are presented in Table $\mathbf{1 .}$

The methods of Experiment 2 were the same as in Experiment 1 with one modification. Experiment 2 used a dual-task RSVP paradigm in which participants were presented with a question about the T1 ("Please identify the second upright word: fat body word, thin body word, household word, or I don't know") and a question about the T2 ("Please identify the first upright word, Piano, Flute, Erhu, or I don't know"). The corresponding response key for each answer was counterbalanced across participants. As in the single-task RSVP in Experiment 1, each participant completed a total of 648 trials of 18 conditions (three categories of $\mathrm{T} 1 \times 6 \mathrm{~T} 1-\mathrm{T} 2$ lags), which were randomly and equally presented in six blocks. Thus, each block consisted of 108 trials with each condition having six trials.

T1 detection accuracy was analyzed using two-way repeated measures ANOVAs (T1 category $\times$ Group). In order to investigate the influence of $\mathrm{BD}$ on $\mathrm{T} 2$ detection, $\mathrm{T} 2$ percent accuracy was analyzed using a 2 (Group: BD vs. Control) $\times 3$
(T1 category: Fatness, Thinness vs Neutral words) $\times 6$ (Lag: Lag 1-6) mixed model ANOVA. In the dual-task RSVP, an individual's mean percentage accuracy for T2 words was calculated for trials where the T1 was identified correctly (Rokke et al., 2002; Fox et al., 2005). Then, for each T1 category, correlations were computed between overall T2 percent accuracy and T2 percent accuracy at each lag with NPS-F and STAI-Trait subscale scores, respectively. Post hoc comparisons for significant main effects and interactions, as well as correlational analysis, was conducted using the Bonferroni correction. For all analyses, $p$-values were corrected for deviations according to the Greenhouse-Geisser correction (Greenhouse and Geisser, 1959).

\section{Results}

\section{Influence of BD on Identification of Different T1s}

A 2 (Group: BD vs. Control group) $\times 3$ (T1 category: Fat, Thin, and Neutral words) ANOVA on correct T1 identification rates showed no significant main effect of Group, $F(1,58)=0.594$, $p=0.444$, partial $\eta^{2}=0.01$; T1 category, $F(2,116)=0.93$, $p=0.368$, partial $\eta^{2}=0.02$; or their interaction, $F(2,116)=1.29$, $p=0.272$, partial $\eta^{2}=0.02$.

\section{Influence of BD on Correct T2 Identification}

A 2 (Group: BD vs. Control group) $\times 3$ (T1 category: Fat, Thin, and Neutral words) $\times 6$ (Lag: Lag1 - Lag6) mixed model ANOVA on T2 percent accuracy revealed a main effect of Lag, $F(5,285)=50.38, p<0.001$, partial $\eta^{2}=0.77$, with lower T2 accuracy at Lags 1 and 2 than at Lags 3-6 (correct identification of the T2 was about $80 \%$ for Lag 1, 86\% for Lag 2, 95\% for Lag 3, 96\% for Lag 4 and Lag 5, and 97\% for Lag 6). Further, the main effect of T1 category was significant, $F(2$, $114)=9.06, p<0.001$, partial $\eta^{2}=0.14$. Post hoc analysis using the Bonferroni correction indicated that participants were generally more accurate in detecting T2 words after neutral T1s $(M=0.93, \mathrm{SE}=0.07)$ than after fat-related $\mathrm{T} 1 \mathrm{~s}(M=0.92$, $\mathrm{SE}=0.01 ; p=0.029)$ or thin-related $\mathrm{T} 1 \mathrm{~s}(M=0.91, \mathrm{SE}=0.01$; $p=0.001)$ but did not differ significantly between fat- and thin-related T1s $(p=0.221)$. There was no significant main effect of Group, $F(1,57)=1.11, p=0.296$, partial $\eta^{2}=0.02$. Notably, the effect of T1 category on T2 accuracy varied as a function of Lag, $F(10,570)=5.28, p<0.001$, partial $\eta 2=0.09$, and this pattern was different between the two groups, as evidenced by the significant Group $\times$ T1 category $\times$ Lag interaction, $F(10,570)=3.08, p=0.004$, partial $\eta^{2}=0.05$ (Figures 2A, 3A).

For a proper interpretation of the three-way interaction, we tested the lag contrasts for each of the T1s separately within each group. For both groups, detection of T2 words after each T1 category was impaired at Lag 1 (all $p s<0.001$ ) and Lag 2 (all ps $<0.016$ ), while these $\mathrm{AB}$ effects rebounded beginning at Lag 3. For the low BD group's performance at Lag 1, participants were less accurate in identifying T2 words after thin-related T1s $(M=0.70, \mathrm{SE}=0.03 ; p<0.001)$ and fat-related T1s $(M=0.75, \mathrm{SE}=0.03 ; p<0.001)$ than after neutral T1s $(M=0.86$, $\mathrm{SE}=0.03$ ), while the high $\mathrm{BD}$ group did not show a significant difference in T2 identification accuracy between the three T1 
TABLE 2 | Correlation matrix between NPS-F and T2 response accuracy rate in Experiment 1 and Experiment 2.

\begin{tabular}{lccccc}
\hline & T-Lag 1 & N-Lag 1 & F-Lag 1 & T-Lag 2 & N-Lag 2 \\
\hline $\begin{array}{l}\text { Experiment 1 } \\
\text { NPS-F }\end{array}$ & $-0.51^{a * * *}$ & -0.21 & $-0.58^{a * * *}$ & $-0.38^{a * *}$ & -0.22 \\
$\begin{array}{l}\text { Experiment 2 } \\
\text { NPS-F }\end{array}$ & $0.43^{a * * *}$ & 0.06 & 0.24 & 0.04 & $-0.29^{*}$ \\
\end{tabular}

NPS-F = negative physical self scale-fatness; STAl-Trait subscale = the state-trait anxiety inventory-trait subscale; $T=$ thin-related T1; $N=$ neutral T1; $F=$ fat-related T1. a significant after Bonferroni correction. ${ }^{*} p<0.05,{ }^{* *} p<0.01$, and ${ }^{* * *} p<0.001$.

categories. Meanwhile, for Lag 1, the low BD group showed lower accuracy in identifying T2 following thin-related $(p=0.007)$ and fat-related T1 words $(p=0.028)$ as compared to the high $\mathrm{BD}$ group (for thin-related $\mathrm{T} 1: M=0.83$, $\mathrm{SE}=0.03$; for fatrelated T1: $M=0.86, \mathrm{SE}=0.03$ ). No other significant simple effects were observed.

\section{Correlation Between BD and T2 Identification}

Correlational analyses were computed across two groups $(n=60)$. Using the Bonferroni correction, correlations were considered significant if the $p$-value was $<0.05 / 18=0.003$. NPS-F scores positively correlated with T2 identification accuracy at Lag 1 following thin-related T1 words $(r=0.43, p<0.001$, Table 2, and Figure 3B). Other correlations were not significant. STAI-Trait subscale scores did not correlate with T2 percent accuracy in any condition (all ps > 0.05).

\section{Discussion}

Experiment 2 investigated the goal-directed attention of bodyrelated stimuli by employing neutral, fat-, and thin-related words as T1s and neutral words as T2s in a dual-task RSVP paradigm. Participants were required to identify both the $\mathrm{T} 1$ and the following T2 as accurately as possible. Significant AB effects were observed among both groups, such that correct detection of T2 after each T1 category was impaired at Lag 1. Notably, the magnitudes of $\mathrm{AB}$ effects following fat- and thin-related $\mathrm{T} 1 \mathrm{~s}$ were significantly smaller in the high BD group than they were in the low $\mathrm{BD}$ group, indicating more effective processing of bodyrelated words in goal-directed attention. These observations are consistent with previous findings in studies using RSVP tasks among participants with anxiety.

\section{GENERAL DISCUSSION}

The present two experiments, employing single- (Experiment 1) and dual-task RSVP (Experiment 2), investigated the attentional interference of fat- and thin-related words in the temporal domain. As expected, participants with BD showed stimulusdriven processing in body-related information. Specifically, in the single-task RSVP (Experiment 1), participants with BD showed spontaneous $\mathrm{AB}$ effects after both fat- and thin-related T1 words. No such effect emerged in the low BD group, suggesting that body-related words automatically capture attention in the high $\mathrm{BD}$ group, even when these words were to-be-ignored stimuli. In the dual-task RSVP (Experiment 2), participants in the low BD group had increased magnitudes of $\mathrm{AB}$ effects following bodyrelated T1 words as compared to neutral T1 words. Participants with $\mathrm{BD}$ did not show this difference. The low $\mathrm{BD}$ group showed lower accuracy in identifying T2 words at Lag 1 following both fat- and thin-related $\mathrm{T} 1$ words as compared to high $\mathrm{BD}$ group, indicating reduced $\mathrm{AB}$ effects after both fat- and thin-related $\mathrm{T} 1$ words among BD group. The findings from Experiment 2 suggest that participants with $\mathrm{BD}$ showed more effective goal-directed attention to body-related information.

\section{Attentional Blink When Body-Related Information Does Not Have to Be Identified}

Experiment 1 investigated stimulus-driven attention to bodyrelated stimuli by employing neutral, fat-, and thin-related T1 in a single-task RSVP paradigm. Participants were required to identify neutral T2s as accurately as possible while ignoring T1s. The observation in Experiment 1 supported the first hypothesis

\section{A}

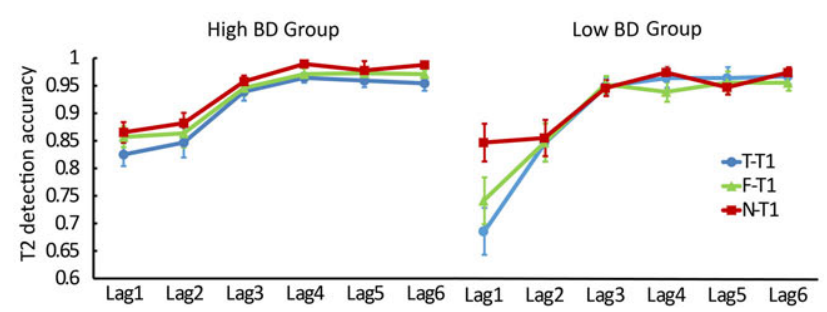

B

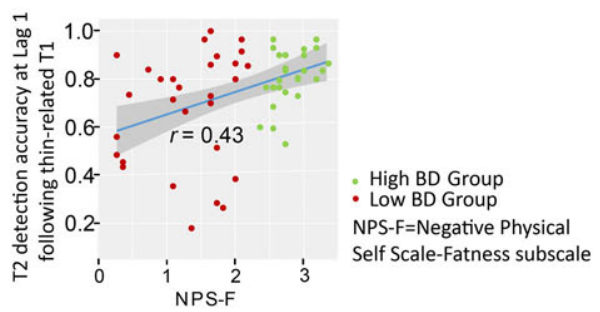

FIGURE 3 | Mean percentage of correct T2 identifications and correlation analysis in dual task RSVP, Experiment 2. (A) Mean percentage of correct T2 identifications as a function of $\mathrm{Tl}$ category and the lag between $\mathrm{Tl}$ and T2. Error bars represent the standard error of the mean. (B) Correlation analysis between NPS-F scores and percentage of correct T2 identifications at Lag 1 following thin-related Tl, with 95\% confidence interval presented. 
that high $\mathrm{BD}$ group showed a spontaneous $\mathrm{AB}$ effect after both fat- and thin-related T1s in the single-task RSVP. Whereas, low $\mathrm{BD}$ group did not show such $\mathrm{AB}$ effects. These observations indicate that participants with high $\mathrm{BD}$ show enhanced stimulusdriven attention to both fat- and thin-related stimuli in the temporal domain, which has also been observed in previous studies using different paradigms. For example, participants with high $\mathrm{BD}$ tend to orient their attention more frequently and more quickly to body-related information as compared to neutral information (Gao et al., 2011). They also allocated more attentional resources to body-related information relative to neutral information (Gao et al., 2014), and perceive vigorous information as negative body-related information (Chen and Jackson, 2005). Correlational analyses found that T2 detection in the short interval after fat- and thin-related T1 words were only negatively correlated with $\mathrm{BD}$, but not anxiety. These observations are particularly important, as they may suggest that alterations in rapid processing of body-related information may be related to BD itself, and not necessarily just anxiety.

One explanation may be that the to-be-ignored body-related information automatically captures attention among individuals with $\mathrm{BD}$, thereby initiate bottom-up processing and resulting in more attentional resources allocated to its consolidation and identification. This would then leave fewer resources available for neutral T2 identification. Results from event-related potential (ERP) studies on emotion-induced blindness gave support to this inference. One previous study reported that larger ABs induced by (to-be-ignored) emotional T1s were associated with an enhanced early posterior negativity (EPN, around $200 \mathrm{~ms}$ ) and late positive potential (LPP, 300-800 ms) ERP component (MacLeod et al., 2017). The EPN has been suggested to represent early semantic activation and selection for further processing in working memory, while the LPP has been suggested to represent the consolidation of a stimulus in working memory (Kennedy and Most, 2015). Future ERP studies are needed to investigate the neutral pattern underlying the current observations in the high BD group.

It is worth noting that the word stimuli in the current study were rated on valence and arousal before the formal experiments. Fat-related words were rated as negative while thinrelated words were rated as positive, and both of them were rated as moderate in arousal. However, the low $\mathrm{BD}$ group did not show a spontaneous $\mathrm{AB}$ effect after body-related $\mathrm{T} 1$ words, which differs from previous observations showing that higharousal or emotional T1 words result in ABs of 200-500 ms, even when they did not need to be identified (for review, see Mishra et al., 2017). These findings may suggest that for people without $\mathrm{BD}$, body shape-related information may be processed differently from emotional stimuli (e.g., fearful, disgusting or happy) that trigger our basic emotions, even though body-related words in the current study were rated as positive or negative with moderate arousal.

\section{Attentional Processing of Body-Related Information When It Has to Be Identified}

In Experiment 2, participants were required to identify both $\mathrm{T} 1$ and T2 words. Fat-, thin-, and neutral words were employed as T1s and neutral words were utilized as T2s. There was no significant difference in $\mathrm{T} 1$ identification regardless of category or the extent of BD.

Consistent with previous studies, we observed poorer performance in T2 identification in a short period after fatand thin-related $\mathrm{T} 1 \mathrm{~s}$ as compared to neutral $\mathrm{T} 1 \mathrm{~s}$ in the low BD group. These observations could be explained, in part, by the two-stage central capacity-limited model (bottleneck theory) of AB (Chun and Potter, 1995). According to this model, processing a target to the level of identification requires two discrete stages: (1) processing and representing of stimulus features (e.g., the perceptual/semantic categorization), which is temporary and fragile, and (2) sustained attention, which is a capacity-limited consolidation stage in working memory and can result in consolidation of stimulus identity sufficient for recognition or report. All the targets in the RSVP stream are encoded and processed up to stage 1 . The $A B$ effects happen during the consolidation stage of $\mathrm{T} 1$ when there are insufficient attentional resources for $\mathrm{T} 2$ identification. Usually, the duration required for T1 consolidation is thought to be around 200$500 \mathrm{~ms}$. Thus, findings from the low BD group suggest that the consolidation stages of fat- and thin-related T1 words recruited more attentional resources than did those of neutral T1s. This would then result in poorer performance on T2 identification in short lags. These enhanced AB effects happened around $106 \mathrm{~ms}$ after a body-related T1 appeared, and it rebounded at Lag 2 around $212 \mathrm{~ms}$.

High BD group, who also showed significant $\mathrm{AB}$ effects in the dual-task RSVP, did not show such difference in AB magnitudes after fat- or thin-related $\mathrm{T} 1$ as compared to those after neutral T1s. The magnitudes of AB effects following fat- and thin-related $\mathrm{T} 1$ words in the high $\mathrm{BD}$ group were significantly smaller than those in the low BD group. These findings are consistent with previous studies investigating temporal processing of emotional information in anxiety (Arend and Botella, 2002; Cisler et al., 2007) and PTSD (Amir et al., 2009). It has been suggested that people with anxiety or PTSD may process disorder-relevant stimuli more rapidly and efficiently as compared to neutral information, resulting in faster recovery of attentional resources after encountering threatening information (Amir et al., 2009). One explanation regarding the reduced $\mathrm{AB}$ effects following body-related $\mathrm{T} 1$ words in high $\mathrm{BD}$ group may be that both fatand thin-related words trigger body-related anxiety or concerns among people with $\mathrm{BD}$. Attentional control processing may be involved to cope with the negative emotion, and thus the consolidation stages for body-related information identification may be shortened implicitly or automatically. These processes among BD group may involve attentional valiance to bodyrelated information in the first stage as well as inhibition of processing that information in the second stage.

\section{BD, EDs, and Anxiety}

The current findings have important clinical implications for understanding body image disturbance or anoxia nervosa (AN). Specifically, the findings among women with BD were consistent with previous observations in participants with high anxiety. For example, Barnard et al. (2005) found that threat-related distractors automatically captured the attention 
of high-anxiety participants and interfered with the following target identification. Arend and Botella (2002) as well as Lystad et al. (2009) manipulated the valence of T1 words and reported that T2 identification accuracy improved following anxiety-related T1s. In these studies, high-anxiety participants enhanced awareness of threat-related stimuli even when this information does not have to be identified, which reduces the accuracy of the following target. Nevertheless, in goaldirected attention, the facilitated consolidation of anxiety-related information among participants with anxiety. Thus, performance on T2 identification in both single- and dual-task RSVP among participants with high $\mathrm{BD}$ or anxiety showed a similar response pattern. Specifically, participants with high BD or anxiety automatically attended to critical stimuli (body- or anxietyrelated stimuli) when the stimuli were designated as distractors, and they processed these stimuli more efficiently than they did neutral targets when the stimuli were employed as targets to identify. On the contrary, the opposite pattern was observed in depression, such that greater $\mathrm{AB}$ effects following negative $\mathrm{T} 1 \mathrm{~s}$ were observed among participants with high depression scores (Koster et al., 2009) or when participants were in a sad mood as compared to a happy mood (Rokke and Lystad, 2015). Likewise, studies focusing on the spatial domain of visual attention have reported similar patterns of attentional bias for $\mathrm{BD}$, EDs, and anxiety disorders (Rieger et al., 1998; Amir et al., 2003; Lee and Shafran, 2004; Gao et al., 2013).

Several investigations have reported high overlap in co-morbidity between anxiety and EDs. One influential study (Lilenfeld et al., 1998) using contemporary familyepidemiological methods to examine patterns of comorbidity and family aggregation of psychiatry disorders for $\mathrm{AN}$ and bulimia nervosa (BN) showed that nearly every AN proband, more than two thirds of $\mathrm{BN}$ probands, and almost half of their relatives had a lifetime history of an anxiety disorder. On the other hand, lifetime rates of major depression disorders were in the low to middle range of those previously reported ED probands and their first-degree relatives. Depression and EDs, thus, appear to be transmitted independently in families. Abundant later work has consistently shown that BD is associated with concurrent overall anxiety symptoms and symptoms of generalized anxiety disorder, panic disorder, social anxiety disorder, and separation anxiety disorder in community samples (Touchette et al., 2011; Cruz-Sáez et al., 2015; Dooley et al., 2015; Duchesne et al., 2017; Vannucci and Ohannessian, 2017).

The above observations suggest that $\mathrm{BD}$ and anxiety may interact tightly with each other, and they may also have very similar visual attentional processing to disturbance-related information. Brain imaging studies have provided evidence to support this inference. The limbic system (especially amygdala and insula) and prefrontal cortex (PFC; medial PFC, and ventrolateral PFC in particular), which is involved in the processing of threat-based stimuli, was found to be actively involved when processing threat-related information among participants with anxiety (Monk et al., 2006, 2008), watching body-related information that could elicit BD among healthy young women (Friederich et al., 2010), among overweight young girls (Gao et al., 2016), when reading negative body-related words among patients with AN (Miyake et al., 2010), as well as when viewing distorted images of themselves among patients with AN (Seeger et al., 2002). Therefore, the cognitive models explaining how attentional bias operates in anxiety could map well onto $\mathrm{BD} / \mathrm{ED}$ s and also may suggest new ideas on how and why attentional bias might develop and function within the context of BD/EDs (for reviews, see Cisler and Koster, 2010; Aspen et al., 2013).

As people with high $\mathrm{BD}$ as well as those with high anxiety show similar attentional processing patterns to disturbance-related information, interventions - namely, attention bias modification $(\mathrm{ABM})$ - aimed at reducing attentional bias and in turn reducing anxiety symptoms among patients with anxiety (MacLeod et al., 2002; Hakamata et al., 2010) should be useful in reducing attentional bias and $\mathrm{BD}$ among participants with $\mathrm{BD}$ or patients with EDs. Indeed, two studies have used the principles of ABM among healthy undergraduate women (Smith and Rieger, 2006; Smeets et al., 2011). However, no consistent observations have emerged yet, and the effects of $\mathrm{ABM}$ in reducing $\mathrm{BD}$ appear to depend on the extent of participants' BD. Thus, in the future, it is critical to address whether $\mathrm{ABM}$ can reduce $\mathrm{BD}$ or increase body esteem via reducing maladaptive attentional bias toward bodyrelated information, and on the other hand, whether participants could benefit from ABM via strengthening adaptive attentional processing of body-related information. Therefore, future studies are warranted to address these questions in diverse populations (e.g., participants with BD, patients with a different kind of EDs).

\section{Limitations and Future Directions}

There were some limitations in the current study. First, this is the first study investigating the moderating effects of BD on bodyrelated stimuli processing in the temporal domain. Therefore, future studies are warranted to reveal the underlying mechanism. For example, by manipulating body-related stimuli as T1, T2, or the background distractors in the RSVP paradigm, it is possible to answer the following questions: (1) whether BD associates with prolonged attention to representations of now-absent visual information and (2) whether people with $\mathrm{BD}$ have a failure to inhibit encoding of non-task body-relevant stimuli. Second, the current sample size was relatively small. Thus, generalization to clinical samples should be undertaken with caution, and the findings should be replicated in larger and more diverse samples. Finally, the fat- and thin-related words used in this study differed both in the content and in the valence. Future research is needed to separate the effects of the content and the valence of the stimuli on the attentional processing.

\section{CONCLUSION}

In conclusion, by employing single- and dual-task RSVP, we provide evidence that $\mathrm{BD}$ is associated with marked differences in the temporal mechanisms of perceptual processing of bodyrelevant information. Young females with BD exhibit robustly enhanced awareness of body-related stimuli, indicating increased recruitment of stimulus-driven processes. In the meantime, 
$\mathrm{BD}$ is associated with facilitated consolidation of body-related information, suggesting more effective processing of body-related information in goal-directed processing.

\section{DATA AVAILABILITY STATEMENT}

The datasets generated for this study are available on request to the corresponding author.

\section{ETHICS STATEMENT}

The studies involving human participants were reviewed and approved by the Ethics Committee of the Psychology Department

\section{REFERENCES}

Amir, N., Elias, J., and Klumpp, H. (2003). Attentional bias to threat in social phobia: facilitated processing of threat or difficulty disengaging attention from threat? Behav. Res. Ther. 41, 1325-1335. doi: 10.1016/s0005-7967(03)00039-1

Amir, N., Taylor, C. T., Bomyea, J. A., and Badour, C. L. (2009). Temporal allocation of attention toward threat in individuals with posttraumatic stress symptoms. J. Anxiety Disord. 23, 1080-1085. doi: 10.1016/j.janxdis.2009.07.010

Annic, A., Bourriez, J. L., Delval, A., Bocquillon, P., Trubert, C., Derambure, P., et al. (2016). Effects of stimulus-driven and goal-directed attention on prepulse inhibition of brain oscillations. Front. Hum. Neurosci. 10:390. doi: 10.3389/ fnhum.2016.00390

Arend, I., and Botella, J. (2002). Emotional stimuli reduce the attentional blink in sub-clinical anxious subjects. Psicothema 14, 209-214.

Aspen, V., Darcy, A. M., and Lock, J. (2013). A review of attention biases in women with eating disorders. Cogn. Emot. 27, 820-838. doi: 10.1080/02699931.2012. 749777

Barnard, P., Ramponi, C., Battye, G., and Mackintosh, B. (2005). Anxiety and the deployment of visual attention over time. Visual Cogn. 12, 181-211. doi: 10.1016/j.jbtep.2018.08.010

Bearman, S. K., Martinez, E., Stice, E., and Presnell, K. (2006). The skinny on body dissatisfaction: a longitudinal study of adolescent girls and boys. J. Youth Adolesc. 35, 217-229. doi: 10.1007/s10964-005-9010-9

Bredemeier, K., Berenbaum, H., Most, S. B., and Simons, D. J. (2011). Links between neuroticism, emotional distress, and disengaging attention: evidence from a single-target RSVP task. Cogn. Emot. 25, 1510-1519. doi: 10.1080/ 02699931.2010 .549460

Browning, M., Holmes, E. A., Murphy, S. E., Goodwin, G. M., and Harmer, C. J. (2010). Lateral prefrontal cortex mediates the cognitive modification of attentional bias. Biol. Psychiatr. 67, 919-925. doi: 10.1016/j.biopsych.2009.10.031

Cash, T., and Labarge, A. (1996). Development of the appearance schemas inventory: a new cognitive body-image assessment. Cogn. Ther. Res. 20, 37-50. doi: $10.1007 / \mathrm{bf} 02229242$

Cash, T. F., and Strachan, M. D. (2002). "Cognitive behavioral approaches to changing body image," in Body Image: a handbook of Theory, Research, and Clinical Practice, eds T. F. Cash, and T. Pruzinsky, (New York, NY: Guilford Press), 478-486.

Chen, H. (2006). The Adolescent Physical Self: theory and Research. Beijing: Xihua Press.

Chen, H., and Jackson, T. (2005). Are cognitive biases associated with body image concerns similar between cultures? Body Image 2, 177-186. doi: 10.1016/j. bodyim.2005.03.005

Chen, H., and Jackson, T. (2007). Stability of body image concerns among Chinese adolescents: nine-month test-retest reliabilities of the negative physical self scale. Percep. Motor Skills 105, 677-680. doi: 10.2466/pms.105.2.677-680

Chen, H., and Jackson, T. (2008). Prevalence and socio demographic correlates of eating disorder endorsements among adolescents and young adults from China. Eur. Eating Disord. Rev. 16, 375-385. doi: 10.1002/erv.837 of Southwest University. The patients/participants provided their written informed consent to participate in this study.

\section{AUTHOR CONTRIBUTIONS}

MS drafted the manuscript. XW analyzed the data and edited the manuscript. XG designed the study and collected the data.

\section{FUNDING}

This work was supported by Central Universities Fundamental Research Funds (\#SWU1809103) to XG.

Chen, H., Jackson, T., and Huang, X. (2006). The negative physical self scale: initial development and validation in samples of Chinese adolescents and young adults. Body Image 3, 401-412. doi: 10.1016/j.bodyim.2006.07.005

Chun, M. M., and Potter, M. C. (1995). A two-stage model for multiple target detection in rapid serial visual presentation. J. Exp. Psychol. 21, 109-127. doi: 10.1037//0096-1523.21.1.109

Cisler, J. M., and Koster, E. W. (2010). Mechanisms of attentional biases towards threat in anxiety disorders: an integrative review. Clin. Psychol. Rev. 30, 203216. doi: 10.1016/j.cpr.2009.11.003

Cisler, J. M., Ries, B. J., and Widner, R. L. Jr. (2007). Examining information processing biases in spider phobia using the rapid serial visual presentation paradigm. J. Anxiety Disord. 21, 977-990. doi: 10.1016/j.janxdis.2006.10.011

Corbetta, M., and Shulman, G. L. (2002). Control of goal-directed and stimulusdriven attention in the brain. Nat. Rev. Neurosci. 3, 201-215. doi: 10.1038/ nrn755

Cruz-Sáez, S., Pascual, A., Salaberria, K., Etxebarria, I., and Echeburúa, E. (2015). Risky eating behaviors and beliefs among adolescent girls. J. Health. Psychol. 20, 154-163. doi: 10.1177/1359105313500683

Davidson, E. J., and Wright, P. (2002). Selective processing of shape and weightrelated words in bulimia nervosa: use of a computerised stroop test. Eating Behav. 3, 261-273. doi: 10.1016/s1471-0153(02)00064-8

de Jong, P. J., Koster, E. W., van Wees, R., and Martens, S. (2010). Angry facial expressions hamper subsequent target identification. Emotion 10, 727-732. doi: $10.1037 / \mathrm{a} 0019353$

Desimone, R., and Duncan, J. (1995). Neural mechanisms of selective visual attention. Annu. Rev. Neurosci. 18, 193-222. doi: 10.1146/annurev.neuro.18. 1.193

Dobson, K. S., and Dozois, D. A. (2004). Attentional biases in eating disorders: a meta-analytic review of Stroop performance. Clin. Psychol. Rev. 23, 1001-1022. doi: 10.1016/j.cpr.2003.09.004

Dooley, B., Fitzgerald, A., and Giollabhui, N. M. (2015). The risk and protective factors associated with depression and anxiety in a national sample of Irish adolescents. Ir. J. Psychol. Med. 32, 93-105. doi: 10.1017/ipm.2014.83

Duchesne, A. P., Dion, J., Lalande, D., Bégin, C., Émond, C., Lalande, G., et al. (2017). Body dissatisfaction and psychological distress in adolescents: is self-esteem a mediator? J. Health Psychol. 22, 1563-1569. doi: 10.1177/ 1359105316631196

Fox, E., Russo, R., and Georgiou, G. A. (2005). Anxiety modulates the degree of attentive resources required to process emotional faces. Cogn. Affect. Behav. Neurosci. 5, 396-404. doi: 10.3758/cabn.5.4.396

Friederich, H. C., Brooks, S., Uher, R., Campbell, I. C., Giampietro, V., Brammer, M., et al. (2010). Neural correlates of body dissatisfaction in anorexia nervosa. Neuropsychologia 48, 2878-2885. doi: 10.1016/j.neuropsychologia.2010. 04.036

Gao, X. (2010). Attentional Bias Among Females with Weight-related Negative Physical Self Schema: Theoretical Model and Processing Rules. Doctor's thesis, Chongqing, Southwest University.

Gao, X., Deng, X., Wen, X., She, Y., Vinke, P. C., and Chen, H. (2016). My body looks like that girl's: body mass index modulates brain activity during 
body Image self-reflection among young women. PLoS One 11:e0164450. doi: 10.1371/journal.pone. 0164450

Gao, X., Deng, X., Yang, J., Liang, S., Liu, J., and Chen, H. (2014). Eyes on the bodies: an eye tracking study on deployment of visual attention among females with body dissatisfaction. Eating Behav. 15, 540-549. doi: 10.1016/j.eatbeh. 2014.08.001

Gao, X., Li, X., Yang, X., Wang, Y., Jackson, T., and Chen, H. (2013). I can't stop looking at them: interactive effects of body mass index and weight dissatisfaction on attention towards body shape photographs. Body Image 10, 191-199. doi: 10.1016/j.bodyim.2012.12.005

Gao, X., Wang, Q., Jackson, T., Zhao, G., Liang, Y., and Chen, H. (2011). Biases in orienting and maintenance of attention among weight dissatisfied women: an eye-movement study. Behav. Res. Ther. 49, 252-259. doi: 10.1016/j.brat.2011. 01.009

Gao, X., Wang, Q. C., Chen, H., Wang, B. Y., and Zhao, G. (2012). Time course of attentional bias components toward body shape related pictures among women with negative physical self image: an eye movement study. Acta Psychol. Sin. 44, 498-510.

Glauert, R., Rhodes, G., Fink, B., and Grammer, K. (2010). Body dissatisfaction and attentional bias to thin bodies. Intern. J. Eating Disord. 43, 42-49. doi: 10.1002/eat.20663

Greenhouse, W. W., and Geisser, S. (1959). On methods in the analysis of profile data. Psychometrika 24, 95-112. doi: 10.1007/bf02289823

Hakamata, Y., Lissek, S., Bar-Haim, Y., Britton, J. C., Fox, N. A., Leibenluft, E., et al. (2010). Attention bias modification treatment: a meta-analysis toward the establishment of novel treatment for anxiety. Biol. Psychiatr. 68, 982-990. doi: 10.1016/j.biopsych.2010.07.021

Kennedy, B. L., and Most, S. B. (2015). The rapid perceptual impact of emotional distractors. PLoS One 1:e0129320. doi: 10.1371/journal.pone.0129320

Koster, E. H. W., De Raedt, R., Verschuere, B., Tibboel, H., and De Jong, P. J. (2009). Negative information enhances the attentional blink in dysphoria. Depress. Anxiety 26, E16-E22. doi: 10.1002/da.20420

Lee, M., and Shafran, R. (2004). Information processing biases in eating disorders. Clin. Psychol. Rev. 24, 215-238. doi: 10.1016/j.cpr.2003.10.004

Lilenfeld, L. R., Kaye, W. H., Greeno, C. G., Merikangas, K. R., Plotnicov, K., Pollice, C., et al. (1998). A controlled family study of anorexia nervosa and bulimia nervosa: psychiatric disorders in first-degree relatives and effects of proband comorbidity. Arch. Gen. Psychiatr. 55, 603-610.

Lystad, C. M., Rokke, P. D., and Stout, D. M. (2009). Emotion congruent facilitation of attention when processing anxious stimuli. Cogn. Ther. Res. 33, 499-510. doi: 10.1007/s10608-008-9210-1

MacLeod, C., Rutherford, E., Campbell, L., Ebsworthy, G., and Holker, L. (2002). Selective attention and emotional vulnerability: assessing the causal basis of their association through the experimental manipulation of attentional bias. J. Abnorm. Psychol. 111, 107-123. doi: 10.1037/0021-843x.111.1.107

MacLeod, J., Stewart, B. M., Newman, A. J., and Arnell, K. M. (2017). Do emotioninduced blindness and the attentional blink share underlying mechanisms? an event-related potential study of emotionally-arousing words. Cogn. Affect. Behav. Neurosci. 17, 592-611. doi: 10.3758/s13415-017-0499-7

Martin, E. W., and Shapiro, K. L. (2008). Does failure to mask T1 cause lag-1 sparing in the attentional blink? Percep. Psychophys. 70, 562-570. doi: 10.3758/ pp.70.3.562

Mathewson, K. J., Arnell, K. M., and Mansfield, C. A. (2008). Capturing and holding attention: the impact of emotional words in rapid serial visual presentation. Mem. Cogn. 36, 182-200. doi: 10.3758/mc.36.1.182

Matthiasdottir, E., Jonsson, S. H., and Kristjansson, A. L. (2010). Body weight dissatisfaction in the Icelandic adult population: a normative discontent? Eur. J. Public Health 22, 116-121. doi: 10.1093/eurpub/ckq178

McHugo, M., Olatunji, B. O., and Zald, D. H. (2013). The emotional attentional blink: what we know so far. Front. Hum. Neurosci. 23:151. doi: 10.3389/fnhum. 2013.00151

Mishra, M. V., Ray, S. B., and Srinivasan, N. (2017). Effect of emotions on temporal attention. Prog. Brain Res. 236, 287-309. doi: 10.1016/bs.pbr.2017.08.014

Miyake, Y., Okamoto, Y., Onoda, K., Shirao, N., Otagaki, Y., and Yamawaki, S. (2010). Neural processing of negative word stimuli concerning body image in patients with eating disorders: an fMRI study. NeuroImage 50, 1333-1339. doi: 10.1016/j.neuroimage.2009.12.095
Monk, C. S., Nelson, E. E., McClure, E. B., Mogg, K., Bradley, B. P., Leibenluft, E., et al. (2006). Ventrolateral prefrontal cortex activation and attentional bias in response to angry faces in adolescents with generalized anxiety disorder. Am. J. Psychiatr. 163, 1091-1097. doi: 10.1176/ajp.2006.163.6.1091

Monk, C. S., Telzer, E. H., Mogg, K., Bradley, B. P., Mai, X., Louro, H. M., et al. (2008). Amygdala and ventrolateral prefrontal cortex activation to masked angry faces in children and adolescents with generalized anxiety disorder. Arch. Gen. Psychiatr. 65, 568-576. doi: 10.1001/archpsyc.65.5.568

Most, S. B., Chun, M. M., Widders, D. M., and Zald, D. H. (2005). Attentional rubbernecking: cognitive control and personality in emotioninduced blindness. Psychon. Bull. Rev. 12, 654-661. doi: 10.3758/bf03196754

Olatunji, B. O., Ciesielski, B. G., Armstrong, T., Zhao, M., and Zald, D. H. (2011). Making something out of nothing: neutral content modulates attention in generalized anxiety disorder. Depress. Anxiety 28, 427-434. doi: 10.1002/da. 20806

Onden-Lim, M., Wu, R., and Grisham, J. (2012). Body image concern and selective attention to disgusting and non-self appearance-related stimuli. Body Image 9, 535-538. doi: 10.1016/j.bodyim.2012.07.005

Perpina, C., Leonard, T., and Treasure, J. (1998). Selective processing of food and body related information and autonomic arousal in patients with eating disorders. Spanish J. Psychol. 1, 3-10. doi: 10.1017/s1138741600005357

Raymond, J. E., Shapiro, K. L., and Arnell, K. M. (1992). Temporary suppression of visual processing in an RSVP task: anattentional blink? J. Exp. Psychol. 18, 849-860. doi: 10.1037//0096-1523.18.3.849

Rieger, E., Schotte, D. E., and Touyz, S. W. (1998). Attentional biases in eating disorders: a visual probe detection procedure. Intern. J. Eating Disord. 23, 199-205. doi: 10.1002/(sici) 1098-108x(199803)23:2<199::aid-eat10>3.3.co;2-o

Rodgers, R. F., and DuBois, R. H. (2016). Cognitive biases to appearance-related stimuli in body dissatisfaction: a systematic review. Clin. Psychol. Rev. 46, 1-11. doi: 10.1016/j.cpr.2016.04.006

Rokke, P. D., Arnell, K. M., Koch, M. D., and Andrews, J. T. (2002). Dualtask attention deficits in dysphoric mood. J. Abnorm. Psychol. 111, 370-379. doi: 10.1037//0021-843x.111.2.370

Rokke, P. D., and Lystad, C. M. (2015). Mood-specific effects in the allocation of attention across time. Cogn. Emot. 29, 27-50. doi: 10.1080/02699931.2014. 893865

Rosser, B., Moss, T., and Rumsey, N. (2010). Attentional and interpretative biases in appearance concern: an investigation of biases in appearance-related information processing. Body Image 7, 251-254. doi: 10.1016/j.bodyim.2010. 02.007

Sackville, T., Schotte, D. E., and Touyz, S. W. (1998). Conscious and preconscious processing of food, body weight and shape, and emotion-related words in women with anorexia nervosa. Intern. J. Eating Disord. 23, 77-82. doi: 10.1002/ (sici) 1098-108x(199801)23:1<77::aid-eat9>3.0.co;2-z

Seeger, G., Braus, D. F., Ruf, M., Goldberger, U., and Schmidt, M. H. (2002). Body image distortion reveals amygdala activation in patients with anorexia nervosa: a functional magnetic resonance imaging study. Neurosci. Lett. 326, 25-28. doi: 10.1016/s0304-3940(02)00312-9

Shapiro, K. L., Caldwell, J., and Sorensen, R. E. (1997). Personal names and the attentional blink: a visual "cocktail party" effect. J. Exp. Psychol. 23, 504-514. doi: 10.1037/0096-1523.23.2.504

Smeets, E., Tiggemann, M., Kemps, E., Mills, J., Hollitt, S., Roefs, A., et al. (2011). Body checking induces an attentional bias for body-related cues. Intern. J. Eating Disord. 44, 50-57. doi: 10.1002/eat.20776

Smith, R. C. (1972). United States. Office of Aviation Medicine. A study of the StateTrait Anxiety Inventory and the assessment of stress under simulated conditions. Washington, DC: Office of Aviation Medicine.

Smith, E., and Rieger, E. (2006). The effect of attentional bias toward shapeand weight-related information on body dissatisfaction. Int. J. Eat. Disord. 39, 509-515. doi: 10.1002/eat.20291

Stice, E., Ng, J., and Shaw, H. (2010). Risk factors and prodromal eating pathology. J. Child Psychol. Psychiatr. 51, 518-525. doi: 10.1111/j.1469-7610.2010.02212.x

Stice, E., and Shaw, H. E. (2002). Role of body dissatisfaction in the onset and maintenance of eating pathology: a synthesis of research findings. J. Psychos. Res. 53, 985-993. doi: 10.1016/s0022-3999(02)00488-9

Touchette, E., Henegar, A., Godart, N. T., Pryor, L., Falissard, B., Tremblay, R. E., et al. (2011). Subclinical eating disorders and their comorbidity with mood 
and anxiety disorders in adolescent girls. Psychiatry Res. 181, 185-192. doi: 10.1016/j.psychres.2010.04.005

Van Dam, N. T., Earleywine, M., and Altarriba, J. (2012). Anxiety attenuates awareness of emotional faces during rapid serial visual presentation. Emotion 12, 796-806. doi: 10.1037/a0024648

Vannucci, A., and Ohannessian, C. M. (2017). Body image dissatisfaction and anxiety trajectories during adolescence. J. Clin. Child Adolesc. Psychol. 47, 785-795. doi: 10.1080/15374416.2017.1390755

Vitousek, K. B., and Hollon, S. D. (1990). The investigation of schematic content and processing in eating disorders. Cogn. Ther. Res. 14, 191-214. doi: 10.1007/ bf01176209

von Lengerke, T., Mielck, A., and Kora Study Group, (2012). Body weight dissatisfaction by socioeconomic status among obese, preobese and normal weight women and men: results of the cross-sectional KORA Augsburg S4 population survey. BMC Public Health 12:342-353. doi: 10.1186/1471-2458$12-342$
Weierich, M. R., and Treat, T. A. (2015). Mechanisms of visual threat detection in specific phobia. Cogn. Emot. 29, 992-100. doi: 10.1080/02699931.2014.960369

Williamson, D. A., White, M. A., York-Crowe, E., and Stewart, T. M. (2004). Cognitive behavioral theories of eating disorders. Behav. Modification 28, 711-738.

Conflict of Interest: The authors declare that the research was conducted in the absence of any commercial or financial relationships that could be construed as a potential conflict of interest.

Copyright (C) 2019 So, Wang and Gao. This is an open-access article distributed under the terms of the Creative Commons Attribution License (CC BY). The use, distribution or reproduction in other forums is permitted, provided the original author(s) and the copyright owner(s) are credited and that the original publication in this journal is cited, in accordance with accepted academic practice. No use, distribution or reproduction is permitted which does not comply with these terms. 


\section{APPENDIX A}

\section{Stimulus Words}

\section{Fat-related words}

肥胖 [Fat (adj.)]

脂肪 [Fat $(\mathrm{n})$.

肥膘 [Excess fat]

膘肉 [Fat meat]

胖子 [Fatty guy]

超重 [Overweight]

肥重 [Heavy]

大象腿 [Elephant's legs]
肥圆 [Round]

鰲肉 [Flabby meat]

肥肥 [Obese]

肉感 [Flashy]

防肿 [Gross]

圆胖 [Plump]

胖妹 [Fat girl]

水桶腰 [Tubby]
Thin-related words

骨感 [Wafer-thin]

纤体 [Lean body]

纤腰 [Thin waist]

细腰 [Slim waist]

纤细 [Slim and narrow]

瘦瘦的 [Slim]

姻娜 [Light and graceful]

杨柳腰 [Willows waist]
纤瘦 [Lean]

苗条 [Thin]

轻盈 [Light]

娇小 [Petite and slim]

窈宛 [Gentle and graceful]

修长 [Slim and tall]

纤身 [Slim body]

曲线美 [Curved] 